\title{
Detección de barreras e implementación de procedimientos para reducir la demora en el tratamiento del síndrome coronario agudo con elevación del segmento ST mediante angioplastia primaria. Experiencia de 20 años de un centro de referencia en una ciudad de alta densidad demográfica
}

\author{
Barriers and Procedures to Reduce Treatment Delay in ST-Segment Elevation Acute Coronary \\ Syndrome with Primary Percutaneous Coronary Intervention. 20-Year Experience of a \\ Tertiary Care Center in a Densely Populated City
}

FEDERICO BLANCO ${ }^{1} \odot$, JORGE SZARFER' ${ }^{1} \oplus$, ALEJANDRO GARCÍA ESCUDERO ${ }^{1}$ RODRIGO BLANCO ${ }^{1}$, FEDERICO ALBORNOZ1',

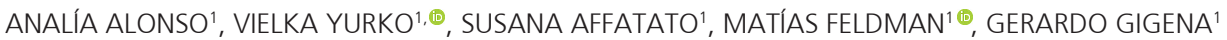

\begin{abstract}
RESUMEN
Introducción: La demora a la reperfusión del síndrome coronario agudo con elevación del segmento ST es un factor determinante en el pronóstico. Su reducción podría disminuir la morbimortalidad.

Objetivo: Identificar y modificar las barreras detectadas en 20 años de tratamiento del síndrome coronario agudo con elevación del segmento ST en un centro de tercer nivel de una ciudad de alta densidad demográfica para evaluar su efecto en el resultado del procedimiento.

Material y métodos: Incluimos prospectiva y consecutivamente del 01/01/2000 al 31/12/2019, 3007 pacientes con síndrome coronario agudo con elevación del segmento ST dentro de las $12 \mathrm{~h}$ de iniciados los síntomas para realizar angioplastia primaria. Se dividió el tiempo desde el comienzo de los síntomas hasta la insuflación del balón en intervalos.

Luego de identificar las barreras (2000-2009) se incorporaron cambios al procedimiento. Se organizó a la población en 2 grupos (G) G1: preimplementación de cambios (2000-2009) y G2: posimplementación (2010-2019).

Resultados: Se incluyeron en G1 1409 pacientes y en G2 1598. Sin diferencias demográficas, excepto por el tipo de angioplastia. Se identificaron demoras al realizar el diagnóstico, de comunicación entre médicos, del traslado y del ingreso del paciente a hemodinamia. Con los cambios, disminuimos el intervalo consulta-contacto con el hemodinamista [G1: 90 min (36-168) vs. G2: 77 $\min (36-144) \mathrm{p}-0,01$ ] y el intervalo contacto hemodinamista-ingreso a Hemodinamia [G1: 75 min (55-100) vs. G2: 51 min (34-70) $\mathrm{p}-0,01]$. Se redujo la mortalidad intrahospitalaria (G1: $9,2 \%$ vs. G2:6,7\% p-0,01) y al 6to mes (G1: $13,1 \%$ vs. G2: $7,5 \% \mathrm{p}-0,01)$. Conclusiones: El retraso al diagnóstico, la dificultad en la comunicación y la forma de traslado fueron las principales causas de demora. La implementación de un protocolo de procedimientos permitió reducir las demoras. La evaluación continua de resultados y la educación permanente, constituyen los pilares fundamentales para la optimización de programas de atención en red.
\end{abstract}

Palabras clave: Síndrome coronario agudo - Infarto del miocardio con elevación del segmento ST - Factores de tiempo - Tiempo para el tratamiento - Angioplastia

\begin{abstract}
Background: The delay to reperfusion of ST-segment elevation acute coronary syndrome (STEACS) is a key factor in its prognosis, and its reduction could reduce morbidity and mortality.

Objective: The aim of this study was to identify and modify the barriers detected in 20 years of STEACS treatment in a tertiary care center of a densely populated city to evaluate their effect on the outcome of the procedure.

Methods: A total of 3007 patients with STEACS within 12 hours of symptoms onset were prospectively and consecutively included to undergo primary percutaneous coronary intervention (PCI) from January 1, 2000 to December 31, 2019. Time from symptoms onset to balloon inflation was divided into intervals. After barriers were identified (2000-2009), the procedure was changed. The population was divided into two groups (G) G1: pre-implementation (2000-2009) and G2: post-implementation (2010-2019) of changes.

Results: G1 included 1409 and G2 1598 patients with no demographic differences except for the type of PCI. Delays were identified in diagnosis, communication between physicians, transfer and admission of the patient to the hemodynamics lab. Procedural changes decreased first medical contact-hemodynamic team contact interval [G1: $90 \mathrm{~min}$ (36-168) vs. G2: 77 min $(36-144) \mathrm{p}-0.01]$ and hemodynamic team contact-hemodynamics lab admission interval [G1: 75 min (55-100) vs. G2: $51 \mathrm{~min}$ (34-70) p-0.01] and reduced in-hospital (G1: 9,2\% vs. G2: 6,7\% p-0,01) and 6-month (G1: 13.1\% vs. G2: 7.5\% p-0. 01) mortality. Conclusions: Delay in diagnosis, difficulty in communication and type of transfer were the most important causes of delay. Implementing a procedural protocol reduced delays. Continuous evaluation of results and permanent education constitute the fundamental cornerstones for optimizing network care programs.
\end{abstract}

Key words: Acute Coronary Syndrome - ST Elevation Myocardial Infarction - Time Factor - Time-to-Treatment - Angioplasty

Rev ARgent CARDiol 2021;89:107-114. http://dx.doi.org/10.7775/rac.es.v89.i2.19989

VER ARTÍCULO RELACIONADO: Rev Argent Cardiol 2021;89:87-88. http://dx.doi.org/10.7775/rac.es.v89.i2.20141

Recibido: 21/11/2020 - Aceptado: 19/01/2021

Dirección para separatas: Dr. Federico Blanco - Hospital General de Agudos “Dr. Cosme Argerich” - División Cardiología - Av. Almirante Brown 240 (C1155ADP) Buenos Aires, Argentina - E-mail: federicoblanco@ymail.com

Este trabajo obtuvo el Premio Dr. Isaac Berconsky 


\begin{tabular}{|c|c|c|c|}
\hline ATCP & Angioplastia primaria & RIC & Rango intercuartil \\
\hline ATCR & Angioplastia de rescate & SCACEST & Síndrome coronario agudo con elevación del segmento ST \\
\hline DE & Desviación estándar & TPB & Intervalo puerta-balón \\
\hline EHU & Equipo de hemodinamia de urgencia & & \\
\hline
\end{tabular}

\section{INTRODUCCIÓN}

El tratamiento de elección en el síndrome coronario agudo con elevación del segmento ST (SCACEST) es la reperfusión, preferentemente mediante angioplastia primaria (ATCP), o con trombolíticos para los pacientes que no tienen acceso a dicha terapéutica. $(1,2)$ En la actualidad, entre un $15 \%$ y un $30 \%$ de los pacientes con criterios de reperfusión no reciben el tratamiento adecuado, y lo que es más preocupante, en menos del $45 \%$ de los pacientes se alcanza la reperfusión dentro de los tiempos recomendados debido a la falta de acceso a un sistema de salud adecuadamente organizado. (3-6) Es necesario destacar dentro de los múltiples factores que determinan la mortalidad al tiempo de isquemia, por su importante efecto en la viabilidad del tejido comprometido y por la posibilidad de reducirlo protocolizando el acceso al tratamiento. Por ello es fundamental analizar y corregir los factores determinantes de las demoras. Está demostrado que para que esto suceda, se debe mejorar la conectividad entre hospitales con distintos niveles de complejidad, mediante la interacción de un servicio de emergencias médicas eficiente, que permita no solo aumentar la proporción de pacientes reperfundidos, sino también reducir las demoras, lo que permite recuperar la función ventricular izquierda y, por lo tanto, disminuir la morbimortalidad. $(7,8)$

Los objetivos de este trabajo son, en primer lugar, presentar los resultados de nuestra experiencia de veinte años trabajando en el tratamiento del SCACEST en la Ciudad de Buenos Aires, divididos en dos etapas: la primera de identificación de barreras y demoras en la reperfusión, y una segunda etapa, luego de la introducción de ciertas modificaciones para superar esas barreras. En segundo lugar, con el análisis de estos datos, realizar nuevos protocolos que permitan mejorar el tratamiento del SCACEST en nuestro ámbito.

\section{MATERIAL Y MÉTODOS}

La población se compone de 3007 pacientes que consultaron a nuestro hospital o derivados por otros hospitales generales del sistema público de la CABA y por algunos derivados de hospitales públicos o privados de la CABA y del Conurbano Bonaerense. Se incluyeron a partir del 1 de enero de 2000 hasta el 31 de diciembre de 2019 en forma prospectiva y consecutiva pacientes con diagnóstico de SCACEST que activaron al servicio de hemodinamia para la realización de ATCP o de rescate (ATCR) dentro de las $12 \mathrm{~h}$ de iniciados los síntomas. Se consideró ATCP la realizada sin administración previa de trombolíticos y ATCR la efectuada de acuerdo con los mismos criterios de admisión, pero con la administración previa de fibrinolíticos ( $100 \%$ estreptocinasa) sin criterios de reperfu- sión. Los datos recabados por el equipo de hemodinamia de urgencia (EHU) se registraron en forma prospectiva a través de un cuestionario diseñado especialmente, se ingresaron y analizaron en una base de datos. Luego de realizar un análisis de los datos en el año $2009(5,6)$ se realizaron una serie de modificaciones con el fin de mejorar los tiempos a la angioplastia (Tabla 1). En nuestro hospital, se conformó un equipo multidisciplinario que involucró diferentes áreas que participan en la atención de los pacientes con SCACEST que incluían a los administrativos responsables de la admisión de los pacientes, los médicos y enfermeros responsables del departamento de urgencias responsable de la guardia externa, los encargados del traslado del paciente (camilleros y auxiliares), los residentes del servicio de cardiología y el EHU (médicos, enfermeros y técnicos) con el fin de optimizar los tiempos al diagnóstico, en el ingreso del paciente al quirófano y en la realización de la angioplastia primaria, con la consecuente retroalimentación periódica de los resultados obtenidos. En los pacientes derivados de otros centros, se mejoró la comunicación con aquellos centros que no cuentan con cardiólogo de guardia, con el fin de ayudar mediante el envío de los electrocardiogramas a través de los smartphones, en el diagnóstico de SCACEST y, por ende, mejorar los tiempos. (9) A su vez, se centralizó la solicitud de la angioplastia mediante el coordinador del sistema público de atención emergencias del Gobierno de la Ciudad de Buenos Aires (SAME), que comenzó a darle prioridad a ese traslado, y en los pacientes que se encontraban estables hemodinámicamente (la mayoría), el traslado comenzó a realizarlo el médico solicitante con la ambulancia del centro referente (hasta el 2009 los pacientes eran trasladados con la unidad coronaria móvil del hospital receptor) (Tabla 1). Dichos cambios se acompañaron con la presentación a las autoridades de la Ciudad de una normativa para optimizar el tratamiento del SCACEST. La población se organizó en dos períodos. El grupo 1 (G1) (preimplementación de las modificaciones) desde el 1 de enero de 2000 hasta el 31 de diciembre de 2009, y el grupo 2 (G2) (posimplementación), desde el 1 de enero de 2010 hasta el 31 de diciembre de 2019. Está de más mencionar que la implementación de las modificaciones llevó cierto tiempo, debido, en muchos casos, a la resistencia de los actores por desconocimiento de las responsabilidades y por ignorancia de la importancia del factor tiempo en el tratamiento del SCACEST.

\section{Variables}

Junto con los datos demográficos y basales de los pacientes, se evaluaron prospectivamente las características clínicas, angiográficas, forma de presentación del infarto, mecanismo de llegada del paciente al sistema de salud (por sus propios medios, en ambulancia del SAME o privada), el domicilio y el tipo de cobertura de los pacientes, la evolución intrahospitalaria y en el seguimiento a 6 meses.

Para el análisis de los tiempos se definió una serie de intervalos temporales (Figura 1):

- Intervalo A (IA): Comprendido desde el inicio de los síntomas hasta la llegada al departamento de urgencias del hospital que activa el sistema.

- Intervalo B (IB): Comprendido entre la llegada al hospital al contacto telefónico con el EHU. 
Tabla 1. Barreras que prolongan el tiempo a la reperfusión (2000-2009) y estrategias implementadas para corregirlas (2010-2019)

\begin{tabular}{|c|c|}
\hline $\begin{array}{l}\text { Barreras que prolongan el tiempo a la } \\
\text { reperfusión }(2000-2009)(5,6)\end{array}$ & Implementación de estrategias (2010- 2019) \\
\hline $\begin{array}{l}\text { Demora en la realización e interpretación } \\
\text { del ECG }\end{array}$ & $\begin{array}{l}\text { Prioridad de atención de pacientes con dolor pre- } \\
\text { cordial en guardia } \\
\text { Envío de ECG por WhatsApp ante duda diagnós- } \\
\text { tica (9) }\end{array}$ \\
\hline $\begin{array}{l}\text { Dificultad en la comunicación con el hemodi- } \\
\text { namista de guardia }\end{array}$ & $\begin{array}{l}\text { Centralización de comunicación entre médicos } \\
\text { mediante la línea del coordinador del SAME }\end{array}$ \\
\hline $\begin{array}{l}\text { Traslado del paciente con ambulancia del } \\
\text { centro receptor }\end{array}$ & $\begin{array}{l}\text { Traslado del paciente con ambulancia del hospital } \\
\text { derivador }\end{array}$ \\
\hline $\begin{array}{l}\text { Demoras en el ingreso al quirófano de he- } \\
\text { modinamia }\end{array}$ & Puenteo de guardia \\
\hline $\begin{array}{l}\text { Demoras en la preparación del paciente y del } \\
\text { quirófano }\end{array}$ & $\begin{array}{l}\text { Preparación del paciente y del quirófano por resi- } \\
\text { dente de cardiología en procedimientos realizados } \\
\text { fuera del horario laboral }\end{array}$ \\
\hline
\end{tabular}

Fig. 1. Intervalos de tiempo analizados.

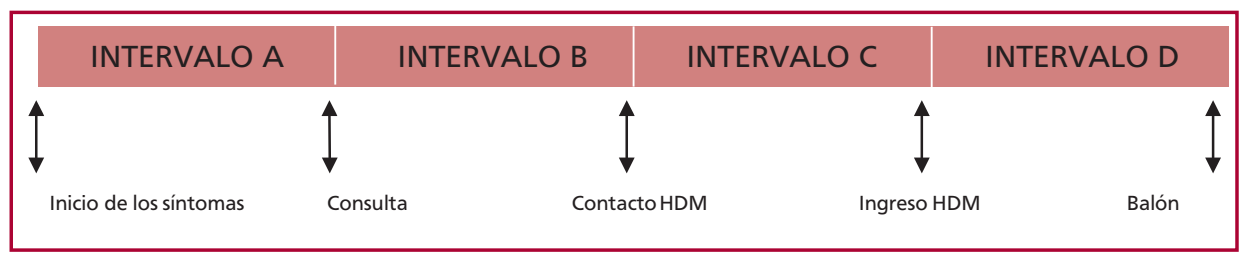

Intervalo A: Desde el inicio de los síntomas hasta la llegada del paciente al departamento de urgencias del hospital que activa el sistema. Intervalo B: Desde la llegada al hospital a la llamada al equipo de hemodinamia (HDM). Intervalo C: Desde el contacto con el equipo de hemodinamia hasta el ingreso del paciente a la sala de hemodinamia. Intervalo D: Desde el ingreso del paciente en la sala de hemodinamia hasta el primer balón insuflado
- Intervalo C (IC): Comprendido entre el contacto con el EHU hasta el ingreso del paciente la sala de hemodinamia.

- Intervalo D (ID): Comprendido entre el ingreso del paciente en la sala de hemodinamia hasta el primer balón insuflado. Con el fin de homogenizar criterios, este intervalo de tiempo en los pacientes referidos desde otro centro constituye el intervalo puerta-balón (TPB).

Se diseñó una ficha donde cada área interviniente registró los horarios. Los distintos tiempos se tomaron de la siguiente forma:

- La hora de inicio de los síntomas y la hora de llegada al hospital directamente del paciente o los familiares.

- La hora de llamada es el momento de contacto telefónico entre el médico que solicita el procedimiento y el EHU.

- El tiempo de ingreso del paciente a la sala de hemodinamia se refiere al momento de primer contacto entre cualquier integrante del EHU y el paciente.

- La hora del primer balón insuflado en la arteria responsable del infarto es registrada por el técnico de hemodinamia por el horario en el angiógrafo.

\section{Análisis estadístico}

Las variables categóricas se expresan como frecuencia y su porcentaje, las variables continúas como medias \pm desviación estándar (DE) o mediana con rango intercuartil (RIC) según su distribución. El análisis de las variables categóricas se realizó con la prueba de chi cuadrado o prueba exacta de Fisher según correspondiera. Las variables continuas fueron analizadas con la prueba de t para 2 colas o Mann-Whitney según su distribución. Se utilizó el software STATA ${ }^{\circledR} 13.0$ y en todos los casos se consideró significativo un valor de $p<0,05$.

\section{Consideraciones éticas}

No fue necesaria la autorización del comité de ética, pues no se difundieron datos personales de los pacientes.

\section{RESULTADOS}

Se incluyeron en total 3007 pacientes (edad media 59 \pm 12 años), el $83 \%$ de sexo masculino. Las características de la población se resumen en la Tabla 2 y solo se encontraron diferencias significativas entre grupos respecto al tipo de angioplastia.

Se observa una elevada prevalencia de hipertensión arterial (57\%), sedentarismo (72\%), tabaquismo (61\%) y diabetes (17\%). Alrededor del $17 \%$ de los pacientes tienen antecedentes de infarto de miocardio y $6 \%$ de insuficiencia cardíaca.

La localización del infarto fue anterior en el $47 \%$ de los casos e inferior en otro $47 \%$. Al ingreso, casi el $80 \%$ de los pacientes presentaba Killip y Kimball A, mientras que la prevalencia de shock cardiogénico fue del $11 \%$. La arteria responsable del SCACEST fue la 
Tabla 2. Características demográficas y angiográficas de los pacientes ingresados

\begin{tabular}{|c|c|c|c|c|}
\hline & $\begin{array}{c}\text { Global } \\
(n=3007)\end{array}$ & $\begin{array}{c}\text { Grupo } 1 \\
(n=1409)\end{array}$ & $\begin{array}{c}\text { Grupo } 2 \\
(n=1598)\end{array}$ & $p$ \\
\hline Sexo masculino $n(\%)$ & $2496(83)$ & $1155(82)$ & $1329(83)$ & NS \\
\hline Edad (años) & $59 \pm 12$ & $59 \pm 12$ & $60 \pm 11$ & NS \\
\hline Hipertensión n (\%) & $1701(57)$ & $774(55)$ & $927(58)$ & NS \\
\hline Dislipemia n (\%) & $1226(41)$ & $602(43)$ & $624(39)$ & NS \\
\hline Tabaquismo n (\%) & $1832(61)$ & $873(62)$ & $959(60)$ & NS \\
\hline Diabetes n (\%) & $519(17)$ & $241(17)$ & 278 (17) & NS \\
\hline Antecedentes heredofamiliares n (\%) & $580(19)$ & $282(29)$ & 298 (19) & NS \\
\hline Sedentarismo n (\%) & $2178(72)$ & $1029(73)$ & $1149(72)$ & NS \\
\hline Antecedentes de infarto de miocardio n (\%) & $511(17)$ & $254(18)$ & $257(16)$ & NS \\
\hline Cirugía de revascularización miocárdica previa n (\%) & $18(0,6)$ & $7(0,5)$ & $11(0,7)$ & NS \\
\hline Antecedentes arteriopatía periférica n (\%) & $136(5)$ & $56(4)$ & $80(5)$ & NS \\
\hline Antecedentes de accidente cerebrovascular n (\%) & $50(1,6)$ & $22(1,5)$ & $28(1,7)$ & NS \\
\hline Antecedentes insuficiencia cardíaca n (\%) & $182(6)$ & $82(6)$ & $100(6)$ & NS \\
\hline Angioplastia primaria $\mathrm{n}(\%)$ & $2743(91)$ & $1235(87)$ & $1508(94)$ & $<0,01$ \\
\hline Angioplastia de rescate $n(\%)$ & $264(9)$ & $174(13)$ & $100(6)$ & $<0,01$ \\
\hline Killip y Kimball A n (\%) & $2360(78)$ & $1103(78)$ & $1257(79)$ & NS \\
\hline Killip y Kimball D n (\%) & $324(11)$ & $158(11)$ & $166(10)$ & NS \\
\hline Infarto de localización anterior n (\%) & $1411(47)$ & $655(46)$ & $756(47)$ & NS \\
\hline Pacientes con domicilio en CABA n (\%) & $1944(65)$ & $897(64)$ & $1047(65)$ & NS \\
\hline Pacientes de Conurbano Bonaerense n (\%) & $1063(35)$ & $512(36)$ & $551(35)$ & NS \\
\hline Pacientes con cobertura médica n (\%) & $1308(43)$ & $593(42)$ & $715(45)$ & NS \\
\hline Pacientes sin cobertura médica n (\%) & $1699(57)$ & $816(58)$ & $883(55)$ & NS \\
\hline Ingreso al hospital por propios medios n (\%) & $1765(59)$ & $835(59)$ & $930(58)$ & NS \\
\hline Ingreso al hospital en ambulancia n (\%) & $1242(41)$ & $574(41)$ & $668(42)$ & NS \\
\hline Consultas a hospital con hemodinamia n (\%) & $1462(49)$ & $674(47)$ & $788(49)$ & NS \\
\hline Derivados desde otro hospital n (\%) & $1545(51)$ & $735(53)$ & $810(51)$ & NS \\
\hline Flujo TIMI 0 basal en la ARI n (\%) & $2368(79)$ & $1106(79)$ & $1262(79)$ & NS \\
\hline Enfermedad de 1 vaso n (\%) & $1578(52)$ & $748(53)$ & $830(52)$ & NS \\
\hline Enfermedad de 2 vasos n (\%) & $808(27)$ & $383(27)$ & $425(27)$ & NS \\
\hline Enfermedad de 3 vasos n (\%) & $487(16)$ & $220(16)$ & $267(17)$ & NS \\
\hline Enfermedad del tronco de la coronaria izquierda & $134(4)$ & $58(4)$ & $76(5)$ & NS \\
\hline
\end{tabular}

ARI: arteria responsable del infarto

descendente anterior en el $46 \%$, coronaria derecha en el $36 \%$ y circunfleja en el $14 \%$. Casi el $80 \%$ de los pacientes presentaban flujo TIMI 0 en la arteria responsable del infarto y el éxito del procedimiento fue mayor al $90 \%$. El 56\% de la población presentaba cobertura médica y el $64 \%$ presentaba domicilio en la Ciudad de Buenos Aires. No existieron diferencias significativas entre grupos respecto a ninguno de estos parámetros (Tabla 2). La demora en la población global desde el inicio de los síntomas hasta la admisión fue de 75 min (RIC 25-75: 30-180) y no hubo diferencias significativas entre grupos. Con los cambios realizados disminuimos significativamente el IB [G1: 90 min (36$168)$ vs. G2:77 $\min (36-144) p<0,01]$ y el IC, tanto al analizar la población global [G1: 65 min (45-115) vs.
G2: 50 min $(28-100) p<0,01]$, los pacientes referidos desde otros hospitales [G1: $75 \mathrm{~min}(55-100)$ vs. G2: 51 min (34-70) $p<0,01]$, como los que consultaron a nuestro centro [G1: $43 \mathrm{~min}$ (23-59) vs. G2: $28 \mathrm{~min}$ (20-60) $p$ 0,01] (Figura 2).

El TPB de los pacientes a los que se les realizó ATCP y que fueron derivados desde otro centro fue de $35 \mathrm{~min}$ (23-55) en el G1 y de 28 min (22-58) en el G2. En el G2, tuvieron un TPB menor de 90 min más del $90 \%$ de los pacientes derivados y un 55\% de los que consultaron a nuestro centro. En este último grupo, el ID fue de 33 min (22-57), por lo que la mayor parte de la demora se encuentra en realizar el diagnóstico y comunicarse con el EHU (Figura 2). Cuando analizamos el porcentaje de pacientes trasladados que alcanzaron el objetivo 


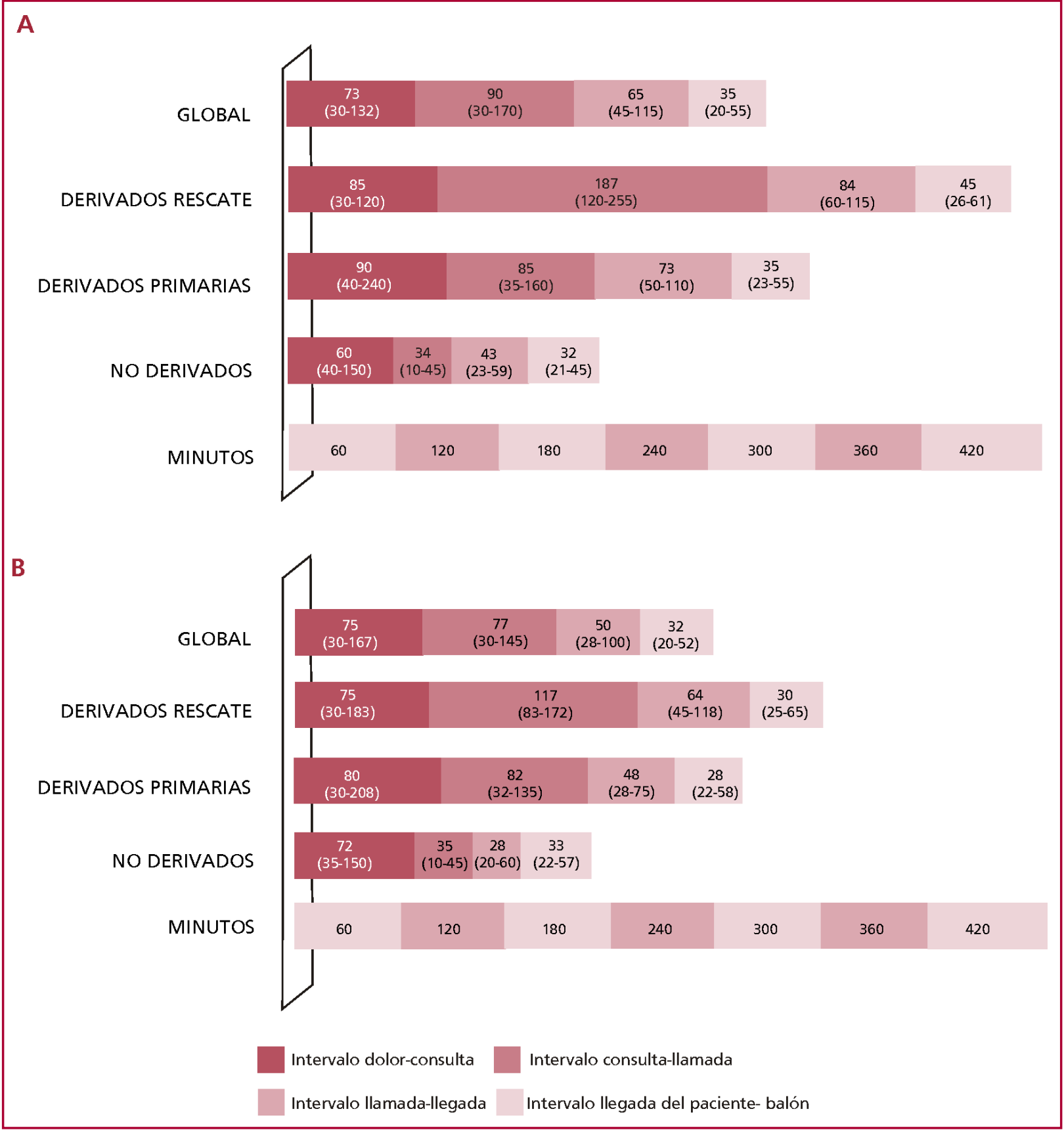

Fig. 2. Intervalos de tiempo analizados en el grupo 1 (A) y en el grupo 2 (B), con las diferentes subpoblaciones de pacientes que conforman cada grupo.

consulta-reperfusión (IB + IC + ID) $<120$ min, ello fue logrado en el 8,9\% de los casos del G1 y en el 21,1\% de los casos en el G2; $(p<0,01)$. También se mejoró en forma significativa el objetivo puerta-puerta (IB + IC), <30 min; (G1: $13,7 \%$ vs. G2: $25,4 \% ; p<0,01$ ). $\mathrm{Al}$ analizar la sobrevida de los pacientes tratados, la misma aumentó significativamente tanto en el ámbito intrahospitalario (G1: $90,8 \%$ vs. G2: $93,3 \% ; p \leq 0,01$ ) como en el seguimiento al 6 mes (G1: 86,9\% vs. G2: $92,5 \% p<0,01$ ) (Figura 3).

\section{DISCUSIÓN}

El SCACEST es una de las patologías que más desafíos impone al sistema de salud. El reconocimiento precoz permite adoptar estrategias para reperfundir el miocardio. Al igual que lo evidenciado en nuestro país, en la ciudad donde nos desempeñamos, la ATCP es la estrategia de reperfusión de elección y, con el correr de los años, fue ganando terreno en detrimento de los fibrinolíticos. (4) También hemos observado una dismi- 


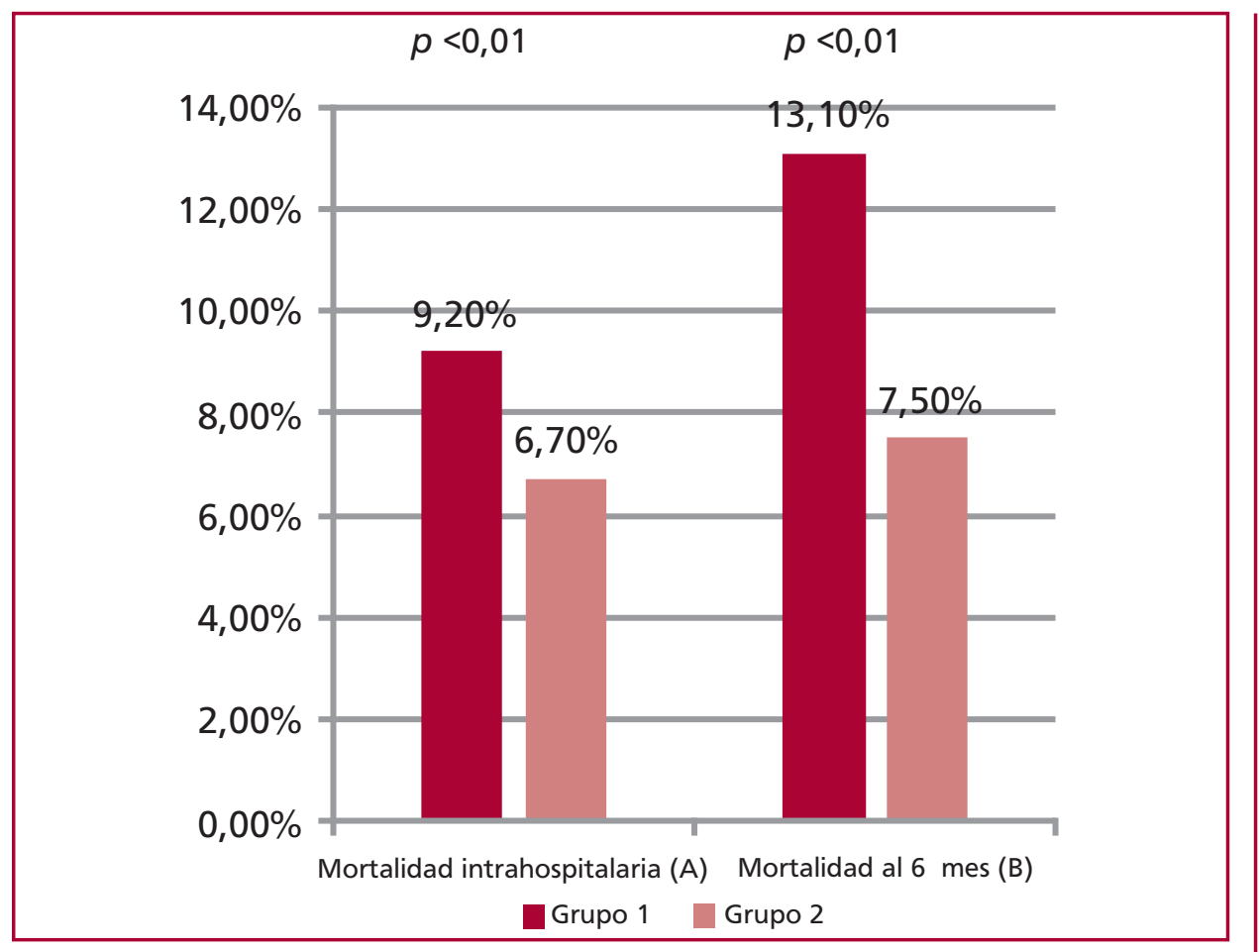

Fig. 3. Mortalidad en el ámbito intrahospitalario $(A)$ y al 6 mes (B) en ambos grupos

nución de la mortalidad intrahospitalaria que, si bien es todavía superior a algunos registros internacionales, es menor que otros registros publicados en nuestro medio. (3, 10-13) Este descenso posiblemente se deba al desarrollo de nuevos fármacos, mayor experiencia del equipo médico tratante, mejor equipamiento, mejores tiempos a la ATCP, lo que se traduce en mejor calidad de atención. Respecto a los tiempos al tratamiento, el conocimiento de las barreras generales y locales permite la instrumentación de medidas correctivas y la evaluación de su impacto en el tiempo. Con las modificaciones implementadas, se observó una reducción del TPB, tanto en pacientes trasladados desde otros centros a nuestro hospital como en aquellos que consultan espontáneamente; nuestro TPB es menor que otros registros de nuestro medio. $(12,14)$. En relación con esto último debe hacerse una aclaración, ya que, en los registros, siempre el TPB es más largo en los pacientes que consultan al hospital con capacidad de hemodinamia respecto de los trasladados desde otro centro, debido a que la preactivación de la sala de hemodinamia disminuye el TPB en este último grupo de pacientes. $(12,15)$ Por esto, en nuestra opinión, en los registros deberían dividirse dichas poblaciones; si se toman ambas en forma global, el TPB dependerá de la prevalencia de pacientes trasladados que presenta el centro, que es más prolongado en los centros con menos porcentaje de pacientes trasladados. (12) También es importante determinar cómo se define el TPB en los diferentes trabajos, ya que hay publicaciones (16) que lo empiezan a medir desde el ingreso del paciente al primer hospital (hospital derivador) y esto último puede llevar a comparaciones erróneas. (14) En nuestra experiencia, hubo una reducción de los tiempos a la angioplastia, luego de realizar modificaciones en el sistema, al igual que lo observado en otros registros de nuestro medio. $(13,15)$ Debido que las ambulancias del SAME no cuentan con la capacidad de realizar electrocardiograma en el domicilio, en muchos casos trasladan al paciente a un hospital sin capacidad de hemodinamia, con lo que se pierde tiempo para el diagnóstico y la preactivación del EHU. Hemos realizado sugerencias a las autoridades con el fin de mejorar este punto. Generalmente, a los pacientes que ingresan al hospital en ambulancia se les realiza más rápido el electrocardiograma diagnóstico, por lo que sería útil concientizar a la población, que cuando una persona presente dolor precordial, se debe llamar al número de emergencias (107 en la Ciudad de Buenos Aires) en lugar de concurrir al hospital. (1, 17-19) También hay que concientizar a los responsables de recibir a los pacientes en las guardias de los hospitales, que los pacientes que consulten por dolor precordial deben tener prioridad en su atención y se les debe realizar un electrocardiograma dentro de los 10 min desde la consulta. (20-22) A su vez, el electrocardiograma debe ser evaluado a la brevedad por el cardiólogo de guardia o, en caso de la institución no cuente con uno, enviarlo en forma remota (smartphone) a algún médico que pueda realizar el diagnóstico de SCACEST. (9, 23-24) En cuanto a los pacientes que se presentan en centros sin capacidad de ATCP, el tiempo transcurrido entre la llegada del paciente al hospital hasta su traslado en ambulancia hacia un centro con capacidad de ATCP, es una medida de calidad asistencial y se recomienda una duración $\leq 30$ min para acelerar el tratamiento de reperfusión. (1, 21, 25) En relación con este punto, la implementación del traslado de los pacientes estables hemodinámicamente 
con ambulancia del hospital derivador, sin necesidad de que sea una ambulancia unidad coronaria móvil, ha permitido acortar el tiempo puerta-puerta. Este traslado rápido es seguro y beneficioso en este grupo de pacientes, tal cual lo demuestran distintos registros. $(26,27)$ Si bien ha mejorado dicho intervalo de tiempo luego de las modificaciones, todavía existen falencias en el sistema de salud de la ciudad donde vivimos. Somos conscientes de que el tratamiento óptimo del SCACEST debe basarse en la utilización de redes entre hospitales con varios niveles de complejidad conectados por un servicio eficiente de ambulancias. $(17,22,28)$ Desde el año 2014 en adelante y con el fin de optimizar la atención del SCACEST de nuestra comunidad, pudimos formar con otros hospitales que también dependen del Gobierno de la Ciudad y tienen la capacidad de realizar ATCP y ATCR en forma ininterrumpida ( $24 \mathrm{~h}$, los 7 d de la semana), una red de hospitales conectados por el SAME, con el fin de optimizar la atención de esta patología. Desde ese año tenemos un registro común de datos, un cuerpo común de definiciones, protocolos de actuación universales en la red, una planificación común y auditoría de resultados. Este tipo de redes reduce los retrasos al tratamiento y aumenta la proporción de pacientes que reciben reperfusión. (22, 29-31) Sabemos que aún tenemos varias cosas que corregir, por lo que consideramos a este trabajo como un impulso para seguir mejorando, con la implementación de iniciativas que reduzcan las demoras, que aumenten el cuidado de los pacientes y, por ende, produzcan un incremento de la calidad de la atención.

\section{Limitaciones}

Se trata de un estudio de limitado alcance debido a que no se puede descartar un sesgo de selección en cuanto al tipo de paciente intervenido. Si bien se incluyeron la totalidad de los pacientes que fueron derivados en dicho intervalo de tiempo al servicio de hemodinamia de nuestro hospital, desconocemos la incidencia real y las características de los infartos tratados mediante fibrinólisis eficaz o no tratados con ninguna terapia de reperfusión en ese período. Por otro lado, si bien se realizaron modificaciones en el sistema, no fue posible llevar a cabo su implementación de un día al otro, por lo que muchos pacientes pertenecientes al G2 presentaron los inconvenientes observados en el G1.

\section{CONCLUSIÓN}

La demora en el diagnóstico, la dificultad en la comunicación y la forma de traslado de los pacientes fueron las principales causas de retraso. La implementación de un nuevo protocolo permitió reducir la demora en la atención de los pacientes con SCACEST. La evaluación continua de resultados, así como la educación permanente del recurso humano médico-asistencial y de la sociedad, constituyen los pilares fundamentales para la optimización de este tipo de programas de atención en red.

\section{Declaración de conflictos de intereses}

Los autores declaran que no poseen conflicto de intereses.

(Véase formulario de conflicto de intereses de los autores en la web / Material suplementario).

\section{BIBLIOGRAFÍA}

1. Ibanez B, James S, Agewall S, Antunes MJ, Bucciarelli-Ducci C, Bueno H, et al. 2017 ESC Guidelines for the management of acute myocardial infarction in patients presenting with ST-segment elevation: the Task Force for the management of acute myocardial infarction in patients presenting with ST-segment elevation of the European Society of Cardiology (ESC). Eur Heart J 2018;39:119-77. http://doi.org/10.1093/eurheartj/ehx393

2. Cambou JP, Simon T, Mulak G, Bataille V, Danchin N. The French registry of Acute ST elevation or non-ST-elevation Myocardial Infarction (FAST-MI): study design and baseline characteristics. Arch Mal Coeur Vaiss 2007;100:524-34. http://doi.org/10.1016/j. acvd.2017.05.001

3. Gagliardi J, Charask A, Perna E, D'Imperio H, Bono J, Castillo Y, et al. Encuesta Nacional de infarto agudo de miocardio con elevación del ST en la República Argentina (ARGEN- IAM-ST). Rev Argent Cardiol 2016;84:548-57.

4. Gagliardi J, Charask A, Higa C, Blanco P, Dini A, Tajer C,et al . Infarto agudo de miocardio en la República Argentina. Análisis comparativo en los últimos 18 años. Resultados de las Encuestas SAC. Rev Argent Cardiol 2007;75:171-8.

5. Blanco F, Riccitelli MA, García Escudero A, Affatato S, Neme R, Gigena G, et al. La demora en la realización de la angioplastia primaria, ¿una causa relacionada con el paciente o con el sistema médico-asistencial? Rev Argent Cardiol 2009;77:14-20.

6. García Escudero A, Riccitelli M, Gaito M, Affatato S, Blanco F, Alonso. et al. Demoras en la realización de la angioplastia primaria en los pacientes trasladados con infarto agudo de miocardio: un problema médico-asistencial. Rev Argent Cardiol 2009;77:88-95.

7. Cequier A, Ariza-Solé A, Elola F, Fernández-Pérez C, Bernal J, Segura J, y cols. Impacto en la mortalidad de diferentes sistemas de asistencia en red para el tratamiento del infarto agudo de miocardio con elevación del segmento ST. La experiencia de España. Rev Esp Cardiol 2017;70:155-61. http://doi.org/10.1016/j.recesp.2016.07.016 8. Szarfer JL, García Escudero A, Gigena G, Affatato S, Riccitelli MA. Angioplastia primaria: Modificaciones de la función ventricular Izquierda. Rev Argent Cardiol 2002;70 (Supl 3) ;110 (resumen)

9. García Escudero A, Blanco R, Blanco F, Gigena G, Szarfer J, Gagliardi J. Smartphones para la transmisión de fotografías digitales de electrocardiogramas en pacientes con síndromes coronarios agudos. Rev Argent Cardiol 2015;83:63-4. http://dx.doi.org/10.7775/ rac.es.v83.i1.3833

10. Danchin N, Popovic B, Puymirat E, Goldstein P, Belle L, Cayla $\mathrm{G}$, et al. Five-year outcomes following timely primary percutaneous intervention, late primary percutaneous intervention, or a pharmacoinvasive strategy in ST-segment elevation myocardial infarction: the FAST-MI programme. Eur Heart J 2020;41:858-66. http://doi. org/10.1093/eurheartj/ehz665.

11. García Aurelio M, Cohen Arazi H, Higa C, Gómez Santa María $\mathrm{H}$, Mauro V, Fernández H, et al. Infarto agudo de miocardio con supradesnivel persistente del segmento ST. Registro multicéntrico SCAR (Síndromes Coronarios Agudos en Argentina) de la Sociedad Argentina de Cardiología. Rev Argent Cardiol 2014; 82:259-67. http:// dx.doi.org/10.7775/rac.es.v82.i4.2169

12. Candiello A, Cigalini I, Zangroniz P, García Escudero A, Ripa L, Lasave L, et al. Iniciativa Stent-Save a Life! Argentina. Rev Argent Cardiol 2019;87:210-6. http://dx.doi.org/10.7775/rac.es.v87.i3.15158 13. Ferrante D, Spolidoro J, Caruso O, Budassi N, Onetto L, Blanco R, et al. Mejora en la reperfusión del infarto de miocardio en Argentina. Rev Argent Salud Pública 2013;4:31-8. 
14. Mariani J, De Abreu M, Tajer C, en representación de los investigadores de la Red para la Atención de los Síndromes Coronarios Agudos. Tiempos y utilización de terapia de reperfusión en un sistema de atención en red. Rev Argent Cardiol 2013;81:233-9. http://dx.doi. org/10.7775/rac.es.v81.i3.1337

15. Nau G, Lalor N, Costabel JP, Pedernera G, Morales P, Candiello A, et al. Optimización del tiempo puerta-balón mediante la implementación de un programa de mejora de procesos. Rev Argent Cardiol 2017;85:118-24. http://dx.doi.org/10.7775/rac.es.v85.i2.9172.

16. Piombo AC, Rolandi F, Fitz Maurice M, Salzberg S, Strumminger M, Zylbersztejn H, et al. Registro de calidad de atención del infarto agudo de miocardio en los hospitales públicos de la ciudad de Buenos Aires. Rev Argent Cardiol 2011;79:132-8.

17. Tra J, van der Wulp I, de Bruijne MC, Wagner C. Exploring the treatment delay in the care of patients with ST-elevation myocardial infarction undergoing acute percutaneous coronary intervention: a cross-sectional study. BMC Health Serv Res 2015;15:340. http://dx.doi. org/10.1186/s12913-015-0993-y.

18. Shavelle DM, Chen A, Matthews R, Roe M, de Lemos J, Jollis J, et al. Predictors of Reperfusion Delay in Patients With ST Elevation Myocardial Infarction Self-Transported to the Hospital (from the American Heart Association's Mission: Lifeline Program). Am J Cardiol 2014;113:798-802. http://dx.doi.org/10.1016/j.amjcard.2013.11.036

19. Bosson N, Baruch T, French W, Fang A, Kaji A, Gausche-Hill M, et al. Regional "Call 911” Emergency Department Protocol to Reduce Interfacility Transfer Delay for Patients With ST-Segment-Elevation Myocardial Infarction. J Am Heart Assoc 2017;6:e006898. http:// dx.doi.org/10.1161/JAHA.117.006898

20. Bagai A, Jollis J, Dauerman H, Peng A, Rokos I, Bates E, et al. Emergency department bypass for ST-Segment-elevation myocardial infarction patients identified with a prehospital electrocardiogram: a report from the American Heart Association Mission: Lifeline program. Circulation 2013;128:352-9. http://dx.doi.org/ 10.1161/ CIRCULATIONAHA.113.002339.

21. Muñoz D, Roettig M, Monk L, Al-Khalidi H, Jollis J , Granger C. Transport time and care processes for patients transferred with ST-segment-elevation myocardial infarction: the reperfusion in acute myocardial infarction in Carolina emergency rooms experience. Circ Cardiovasc Interv 2012;5:555-62. http://dx.doi.org/10.1161/CIRCINTERVENTIONS.112.968461

22. Jollis JG, Al-Khalidi HR, Roettig ML, Berger P, Corbett C, Doerfler $\mathrm{S}$, e al. Impact of Regionalization of ST-Segment-Elevation Myocardial Infarction Care on Treatment Times and Outcomes for Emergency Medical Services-Transported Patients Presenting to Hospitals With Percutaneous Coronary Intervention: Mission: Lifeline Accelerator-2.
Circulation 2018;137:376-87. http://dx.doi.org/10.1161/CIRCULATIONAHA.117.032446.

23. Mahmoud K, Gu L, Nijsten M, de Vos R, Niewland W, Zijlstra F, et al. Interhospital transfer due to failed prehospital diagnosis for primary percutaneous coronary intervention: an observational study on incidence, predictors, and clinical impact. Eur Heart J Acute Cardiovasc Care 2013;2:166-75. http://dx.doi.org/10.1177/2048872613481449.

24. Yu SH, Shih HM, Chang S, Chen WK, Li CY. Social media communication shorten door-to-balloon time in patients with STelevation myocardial infarction. Medicine 2019;98:e14791. http:// dx.doi.org/10.1097/MD.0000000000014791.

25. Wang TY, Nallamothu BK, Krumholz HM, Li S, Roe MT, Jollis JG, et al. Association of door-in to door-out time with reperfusion delays and outcomes among patients transferred for primary percutaneous coronary intervention. JAMA 2011;305:2540-7. http://dx.doi. org/10.1001/jama.2011.862.

26. Shi O, Khan A, Rezai M, Jackevicius C, Cox J, Atzema C, et al. Factors associated with door-in to door-out delays among ST-segment elevation myocardial infarction (STEMI) patients transferred for primary percutaneous coronary intervention: a population-based cohort study in Ontario, Canada. BMC Cardiovasc Disord 2018;18:204. http:// dx.doi.org/10.1186/s12872-018-0940-z.

27. Cassan S, Rata M, Vallenet C, Fromage P, Champly F, Broin P et al. Early inter-hospital transfer of patients with myocardial infarction without a doctor, paramedic or nurse on board: results from a French regional emergency care network. BMC Emerg Med 2019 19:60. http://dx.doi.org/10.1186/s12873-019-0280-z.

28. Loh J, Satler L, Pendyala L, Minha S, Frohna W, Torguson R, et al. Use of emergency medical services expedites in-hospital care processes in patients presenting with ST-segment elevation myocardial infarction undergoing primary percutaneous coronary intervention. Cardiovasc Revasc Med 2014;15:219-25. http://dx.doi.org/10.1016/j.carrev.2014.03.011 29. Kalla K, Christ G, Karnik R, Malzer R, Norman G, Prachar H, et al. Implementation of guidelines improves the standard of care: the Viennese registry on reperfusion strategies in ST-elevation myocardial infarction (Vienna STEMI Registry). Circulation 2006;113:2398-405. http://dx.doi.org/10.1161/CIRCULATIONAHA.105.586198

30. Henry TD, Sharkey SW, Burke MN, Chavez IJ, Graham KJ, Henry $\mathrm{CR}$, et al. A regional system to provide timely access to percutaneous coronary intervention for ST-elevation myocardial infarction. Circulation 2007;116:721-8. http://dx.doi.org/10.1161/CIRCULATIONAHA.107.694141.

31. Le May MR, So DY, Dionne R, Glover CA, Froeschl MP, Wells GA, et al. A citywide protocol for primary PCI in STsegment elevation myocardial infarction. N Engl J Med 2008; 358:231-40.http://dx.doi. org/10.1056/NEJMoa073102 